Supporting Information

\title{
Global extraction from parallel reaction monitoring to quantify background peptides for improved normalization and quality control in targeted proteomics
}

\author{
Andrew G. Chambers ${ }^{1}$, Steve M. M. Sweet ${ }^{1}$, David Chain ${ }^{2}$, Yeoun Jin Kim ${ }^{1 *}$ \\ ${ }^{1}$ Translational Medicine, Oncology R\&D, and ${ }^{2}$ Clinical Pharmacology \& Safety Sciences, \\ BioPharmaceuticals R\&D, AstraZeneca, Gaithersburg, Maryland
}

\author{
*Corresponding author: \\ Yeoun Jin Kim \\ One MedImmune Way, Gaithersburg, MD 20878 \\ Phone: 301-398-2736 \\ Email: yeounjin.kim@astrazeneca.com
}

Supplementary Figures: Table of Contents

Figure S1. Comparison of the unscheduled and scheduled PRM methods used to monitor the 30 transitions (light and heavy) for the 15 peptides of interest. The scheduled method also included an additional 418 extra transitions to simulate a highly multiplexed assay. Both methods monitored approximately 30 transitions for a single acquisition cycle.

Figure S2. Histograms for the number of precursors quantified at precursor $\mathrm{m} / \mathrm{z}$ values for DDA, DIA, unscheduled PRM, and scheduled PRM methods. A single acquisition of $500 \mathrm{ng}$ of a HeLa cell digest is shown for each analysis. All raw data files were directly analyzed by MaxQuant and Spectronaut.

Figure S3. Background precursors quantified from $500 \mathrm{ng}$ of a HeLa cell digest by PRM methods, using different precursor isolation windows. (A) Reproducibility of precursor quantification; (B) precursor intensity variation (outliers are not shown for clarity).

Figure S4. Sample normalization factors for samples containing 350, 425, 500, 575, or 650 ng of total HeLa cell digest, representing a $-30 \%,-15 \%, 0 \%,+15 \%$, or $+30 \%$ deviation in input sample material. These samples were prepared and analyzed in triplicate $(n=15)$ for both unscheduled and scheduled PRM. The untargeted peptides were quantified by MaxQuant or Spectronaut analysis. The sample normalization factors were calculated by inverse of the sample median precursor intensity divided by the median of the sample median precursor intensity, as shown in Table S3.

Figure S5. Unscheduled PRM analysis of 5 patient-derived xenograft tissue samples. Selected ion chromatograms are shown for AFVDFLSEIK (targeted) and the missed cleavage form AFVDFLSEIKEER (untargeted). The endogenous peptide chromatograms as extracted by 
Spectronaut are shown. The variation in the raw peak area ratio across samples may suggest variable tryptic cleavage efficiency at this lysine residue. 
A

Unscheduled PRM

$\because$ targeted

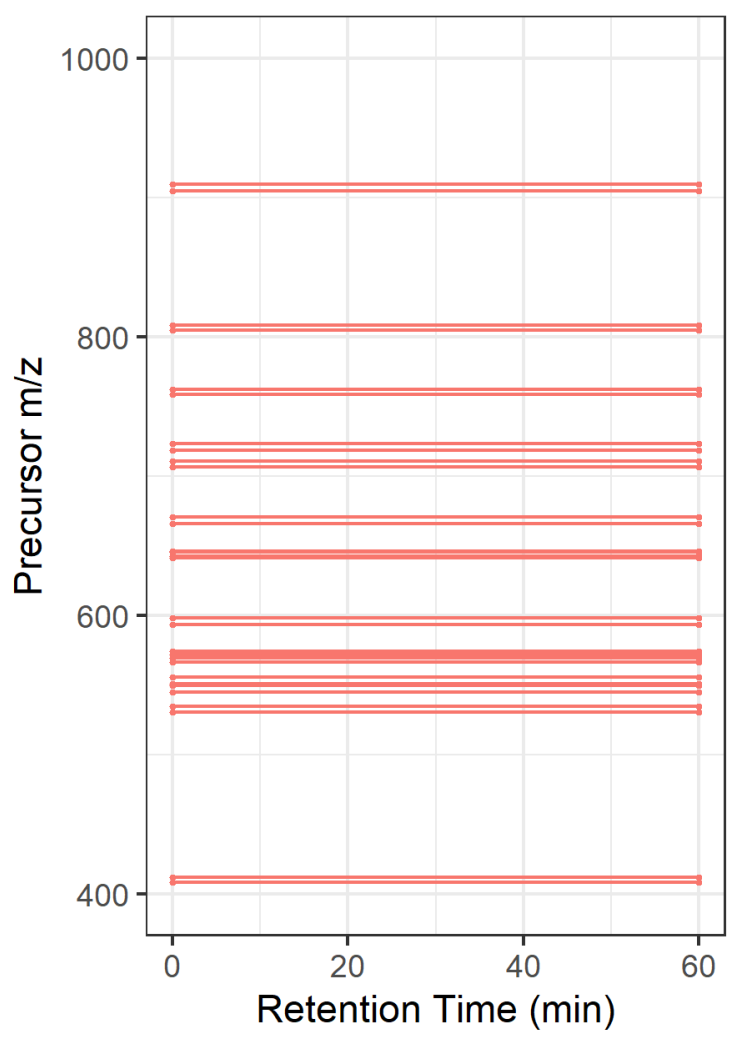

B Scheduled PRM

$\longrightarrow$ targeted $\longrightarrow$ extra

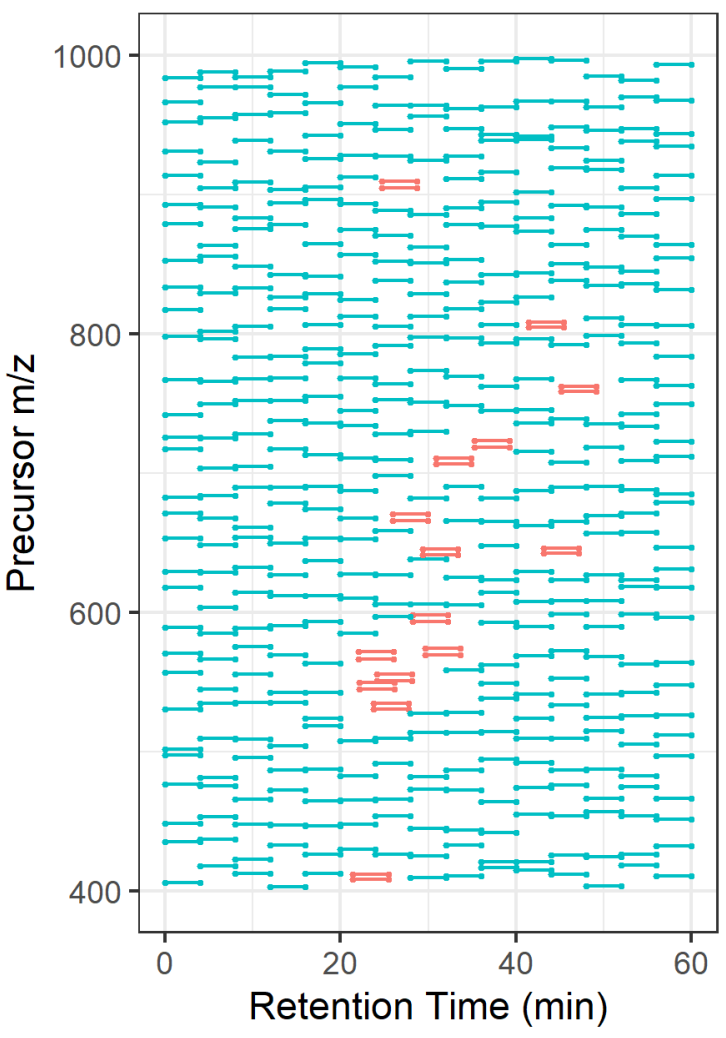

Figure S1. Comparison of the unscheduled and scheduled PRM methods used to monitor the 30 transitions (light and heavy) for the 15 peptides of interest. The scheduled method also included an additional 418 extra transitions to simulate a highly multiplexed assay. Both methods monitored approximately 30 transitions for a single acquisition cycle. 


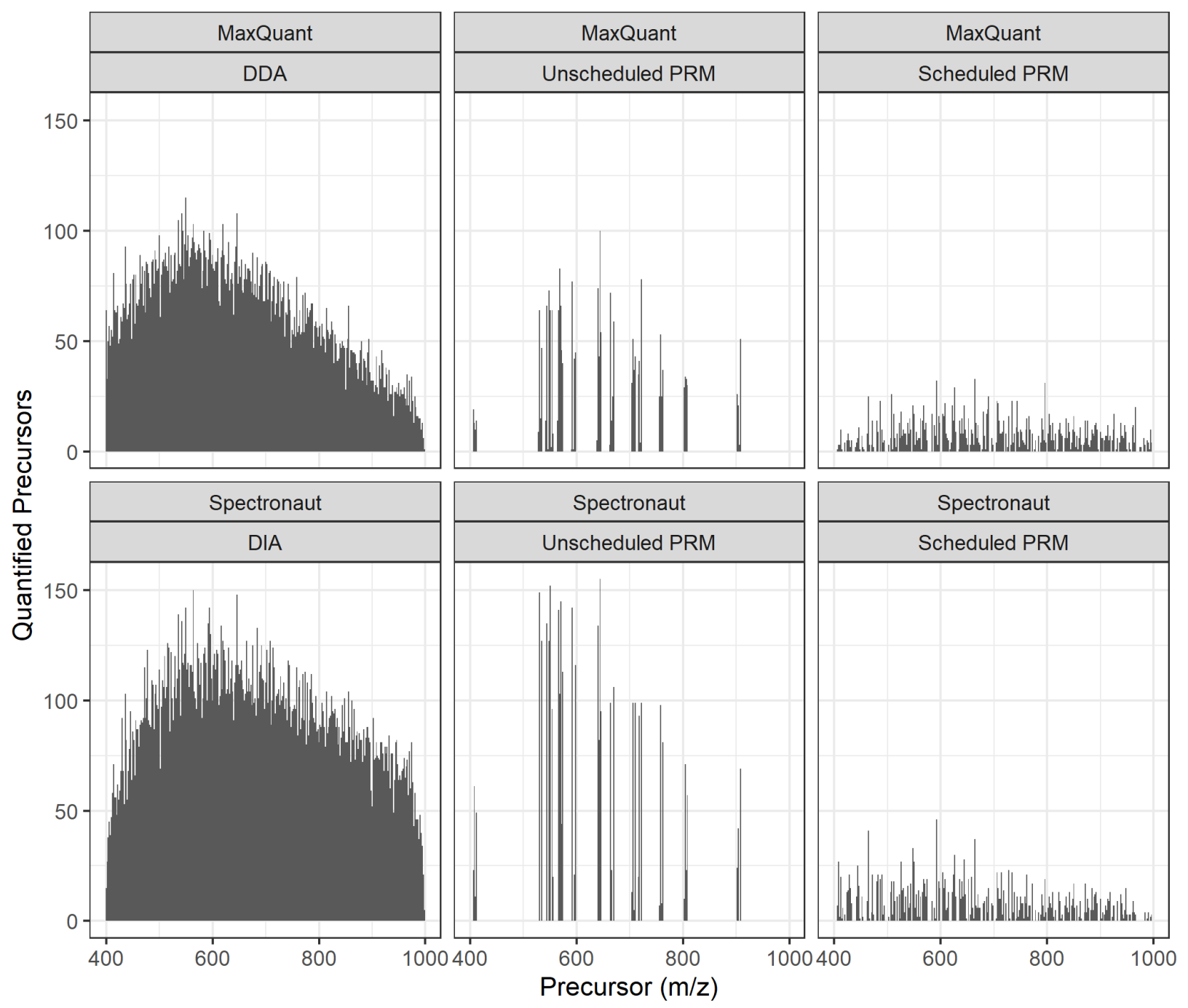

Figure S2. Histograms for the number of precursors quantified at precursor $\mathrm{m} / \mathrm{z}$ values for DDA, DIA, unscheduled PRM, and scheduled PRM methods. A single acquisition of $500 \mathrm{ng}$ of a HeLa cell digest is shown for each analysis. All raw data files were directly analyzed by MaxQuant and Spectronaut. 
A

Precursor Detection Frequency ( $=3$ LC-MS runs)
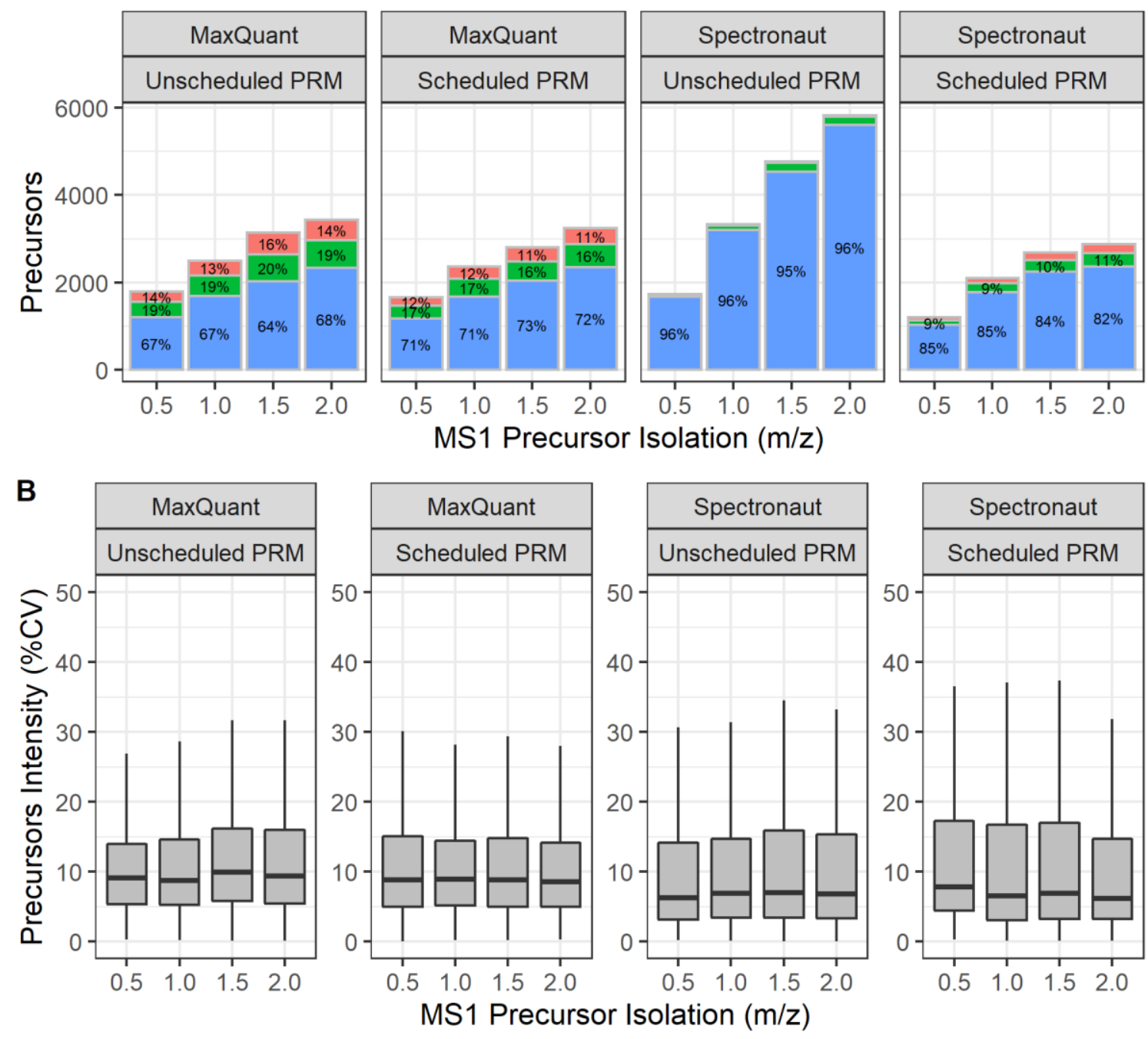

Figure S3. Background precursors quantified from $500 \mathrm{ng}$ of a HeLa cell digest by PRM methods, using different precursor isolation windows. (A) Reproducibility of precursor quantification; (B) precursor intensity variation (outliers are not shown for clarity). 


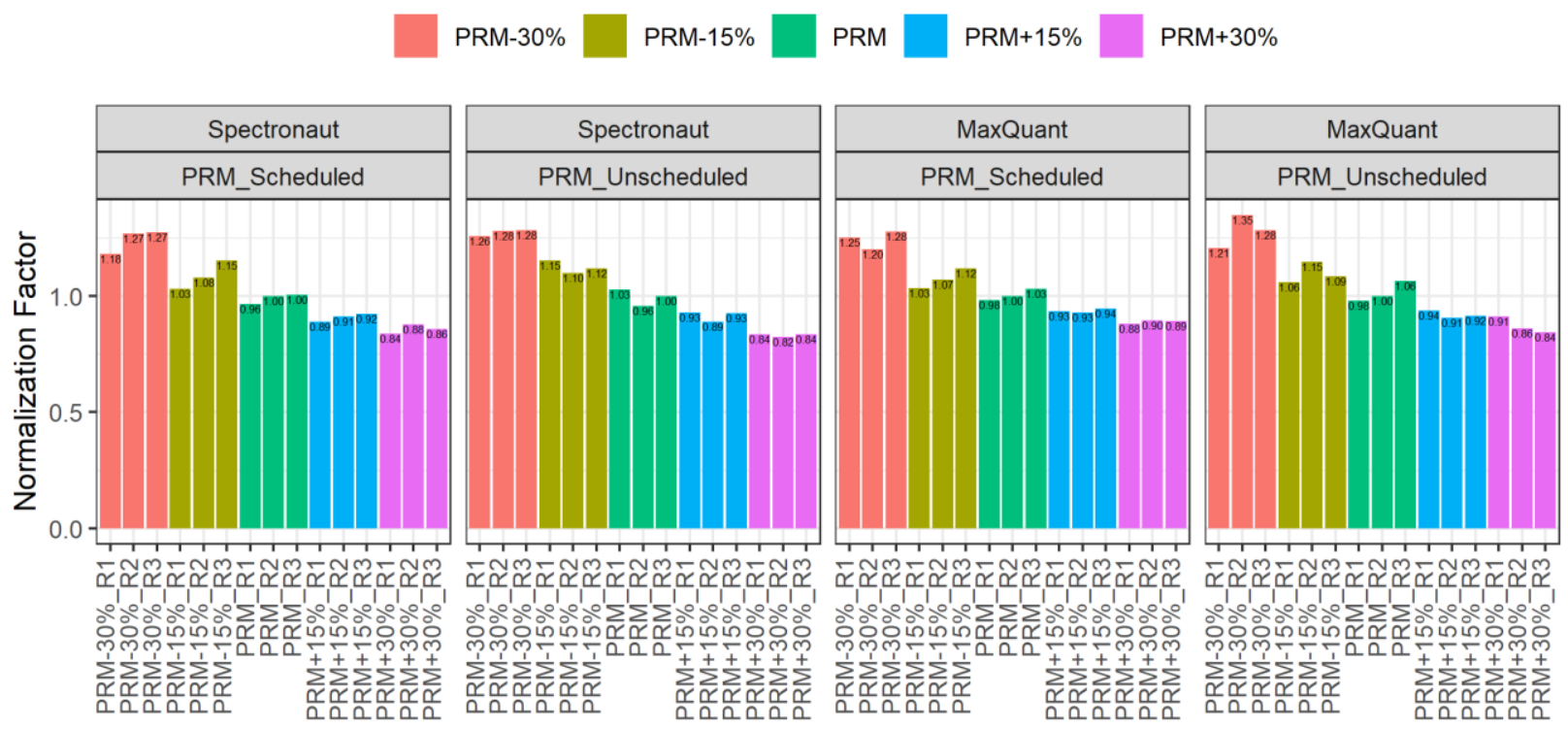

Figure S4. Samples containing 350, 425, 500, 575, or $650 \mathrm{ng}$ of total HeLa cell digest, representing a $-30 \%,-15 \%, 0 \%,+15 \%$, or $+30 \%$ deviation. These samples were prepared and analyzed in triplicate $(n=15)$ for both unscheduled and scheduled PRM. The untargeted peptides were quantified by MaxQuant or Spectronaut analysis. The sample normalization factors were calculated by inverse of the sample median precursor intensity divided by the median of the sample median precursor intensity, as shown in Table S3. 

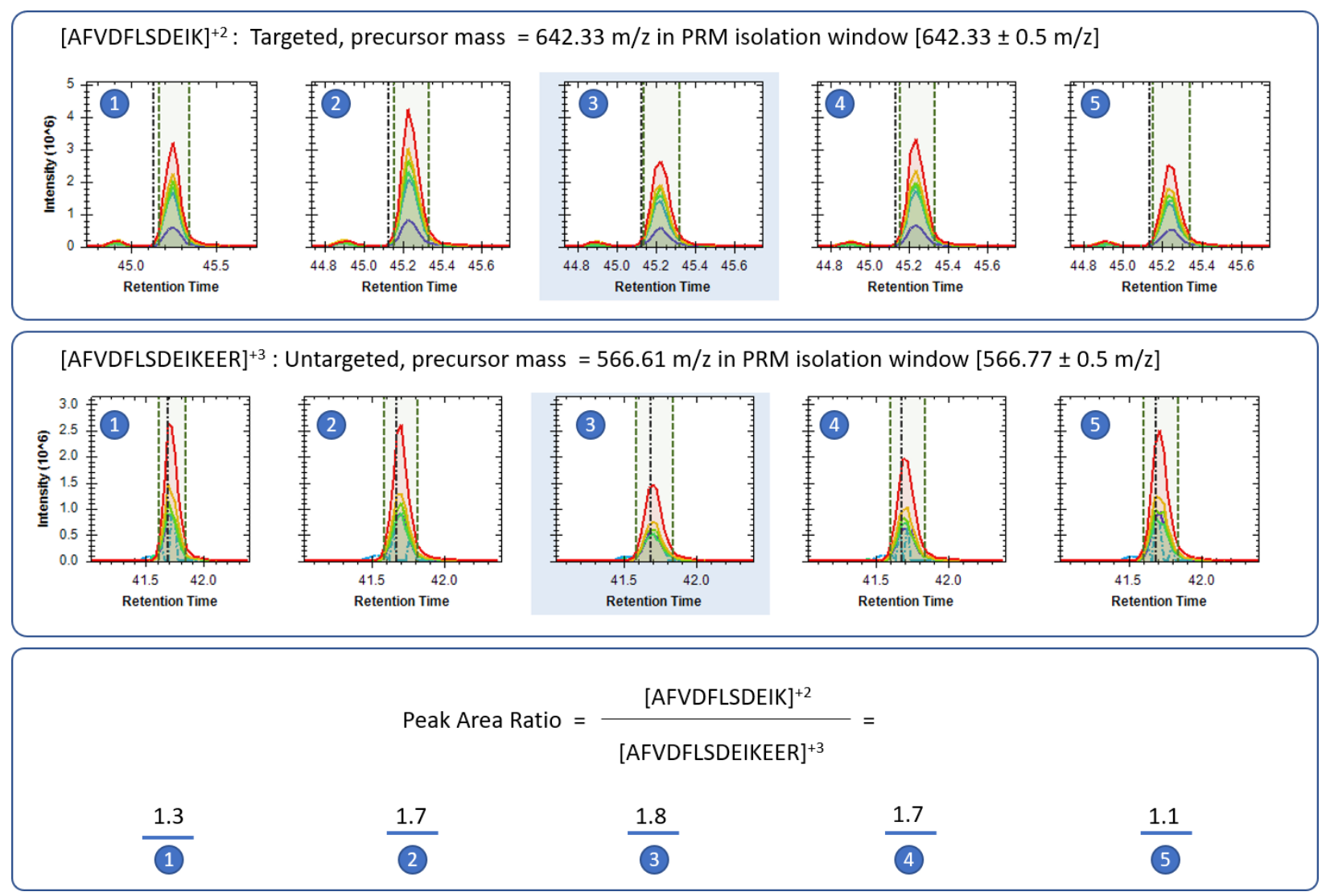

Figure S5. Unscheduled PRM analysis of 5 patient-derived xenograft tissue samples. Selected ion chromatograms are shown for AFVDFLSEIK (targeted) and the missed cleavage form AFVDFLSEIKEER (untargeted). The endogenous peptide chromatograms as extracted by Spectronaut are shown. The variation in the raw peak area ratio across samples may suggest variable tryptic cleavage efficiency at this lysine residue. 